\title{
Alternative Approach to Hybrid Powered Vehicles
}

\author{
G. Sai Kiran ${ }^{1}$, Dr. S Madhava Reddy ${ }^{2}$ \\ ${ }^{\text {I}}$ (UG Scholar, Department of Mechatronics, Mahatma Gandhi Institute OF Technology, Gandipet, Hyderabad, \\ India) \\ ${ }_{2}^{2}$ (Professor, Department of Mechatronics, Mahatma Gandhi Institute OF Technology, Gandipet, Hyderabad, \\ India)
}

\begin{abstract}
This paper presents an alternative approach to conservative hybrid technology which is currently used in automobiles. Hybrid technology uses traditional engine in company with generator set to increase the efficiency from internal combustion(IC) vehicles, but alternatively the flywheels can be slightly modified to extract the energy and storing it in the battery (acting as a generator) while driving the automobile and later using this energy to drive the flywheel, thus powering the vehicle. This way of powering the vehicle by two different sources can increase the efficiency and making the automobile more environmentally friendly. In this substitute approach the flywheel of the engine can itself be used as a secondary power source, with necessary modifications, as a brushless DC motor/generator thus increasing the efficiency while decreasing the overall weight of the engine.
\end{abstract}

Keywords: Brushless DC Motor/Generator, Efficiency, Environmental friendly, Flywheels, Hybrid technology.

\section{Introduction}

The world today uses enormous transport facilities for various purposes and its utilization has been increased since late 1900's. It is estimated that in India alone 182 million vehicles are registered in 2013 and drove more than 3 trillion miles every year. By far the largest share is consumed by cars, light trucks, and motorcycles-about $58 \%$ in 2013 , followed by other trucks (23\%), aircraft (8\%), boats and ships (4\%), and trains and buses (3\%). In order to account for growing needs of the world, various advancements have been made in automobile fuel consumption.

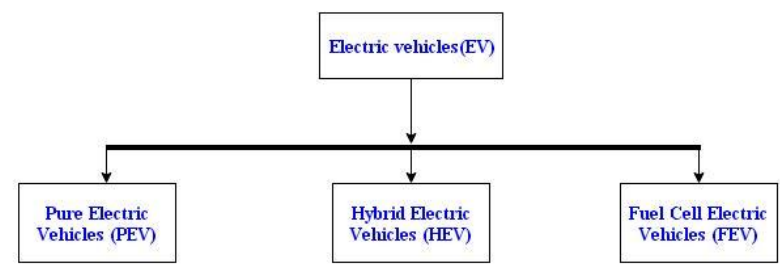

Fig.1 Classification of Electric vehicles

A particular advancement is the use of hybrid powered vehicles. According to technical Committee 69 (electric road vehicles) of the International Electro technical Commission, a Hybrid Electric Vehicle (HEV) is a vehicle in which propulsion energy is available from two or more kinds or types of energy sources or converters, and at least one of them can deliver electrical energy. From the above definition HEV uses an internal combustion engine and can be fueled like normal cars but have an electric motor and battery, and can be partially or wholly powered by electricity. By using both a conventional engine and electric motor, the best hybrids attain significantly better fuel efficiency than their non-hybrid counterparts. They also release less pollutant into atmosphere and account for fuel savings.

A traditional vehicle has a mechanical drive train that includes combustion engine, fuel tank, gear box, transmission mechanism connected to wheels. HEV has two drive trains, mechanical and electrical. Mechanical drive contains identical parts as ordinary vehicles. Electric drive includes battery, an electric motor, and power electronics for control. These two drive trains are interconnected with each other and share some components like transmission, differential, gear box. Electric drive train can handle a wide variety of speeds and loads without losing efficiency. 


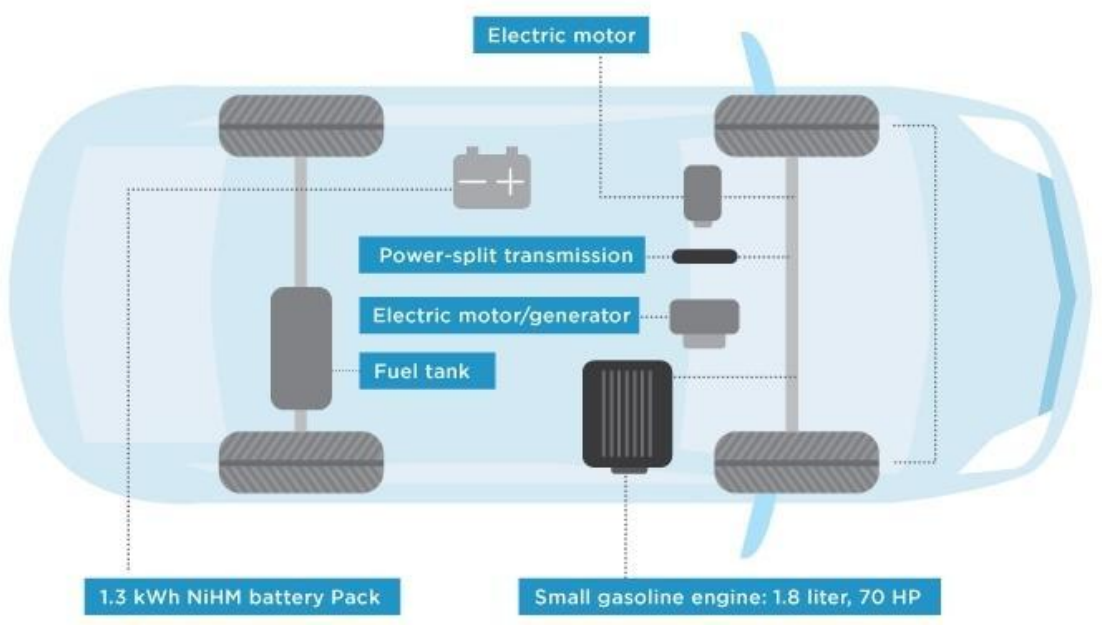

Fig.2 Line sketch of Hybrid vehicles

\section{Various Hybrid Electric Vehicles Configurations:}

1. Parallel Hybrids: In parallel hybrids, the internal combustion engine and the electric motor are both coupled to the mechanical transmission system and can simultaneously transmit power to drive the wheels, usually through a usual transmission. The internal combustion engine of various parallel hybrids can also act as a generator for supplemental recharging. Currently, these commercialized hybrids use a full size combustion engine with a single, small electric motor designed to adjunct the main engine, not to be the sole source of motive power from launch. Parallel hybrids are more efficient than their non-hybrid counterpart vehicles especially during urban stop-and-go conditions.

2. Series Hybrids: In series hybrids only the electric motor drives the drive train, and a smaller internal combustion engine works as a generator to power the electric motor or to charge the batteries. They also usually have a larger battery pack than parallel hybrids, making them more expensive. When the batteries are low, the small IC engine can generate power, making them more efficient.

3. Power-split Hybrids: These hybrids take the advantage of a combination of series and parallel attributes. This result in efficient functioning of the vehicle, since series hybrids tend to be more productive at lower speeds and parallel hybrids tend to be more efficient at higher speeds, consequently these require higher investment.

4. Plug-in Hybrids: The most advanced hybrids have larger battery packs and can recharge their batteries from an outlet, allowing them to drive extended distances on electricity before switching to their standard fuel. These hybrids are known as "plug-in hybrids", these cars can offer much-improved environmental performance and work efficiently thus increasing fuel savings by substituting grid electricity for gasoline or other fuels. Also these hybrids allow some of that energy to be captured, turned into electricity, and stored in the batteries. This stored energy can later be used to run the motor and accelerate the vehicle.

The commercial available HEV uses the following advantageous technology during operation.

a. Regenerative Braking: The resistance to the drive train is offered by the motor causing the wheels to slow down. In addition, the wheel drives the motor causing it to act as a generator and store the output energy in the batteries.

b. Motor Assist IC Engine: The electric motor assist internal combustion engine during acceleration to speed up the vehicle. This causes the efficient mode of operation of IC engine and account for low fuel consumption.

c. Auto Start/Shut down: The motor in HEVs automatically start and assist in driving and automatically shuts down when it is not in use.

From the above depictions it is clear that hybrid vehicles use a separate motor/generator for capturing the energy from the crankshaft and convert into the electrical energy, storing it in the battery. This addition of separate motor/generator to the conventional automobile increases the weight of the vehicle and makes a difference to the efficiency of the automobile. The weight of an automobile decides to greater extent the efficiency. The improved design of the hybrid vehicle has been discussed in the subsequent sections. 


\section{Principle of Operation}

The simple principle involved in redesigning a hybrid vehicle can be best understood from the working of a brushless DC motor (BLDC motor). BLDC motor consists of a magnetic rotor and stator with winding pairs that can be independently energized by detecting the angle of the shaft. Hall Effect sensors are used for the purpose of determining the angular position of the shaft. The signal from the sensors is used by electronic commutator to drive the current in succeeding windings thus energizing that winding pair, in such a way that it attracts the rotor magnet. Thus with the help of attractive forces generated between the permanent magnet and stator windings, the BLDC motor can be held to make turning motion.

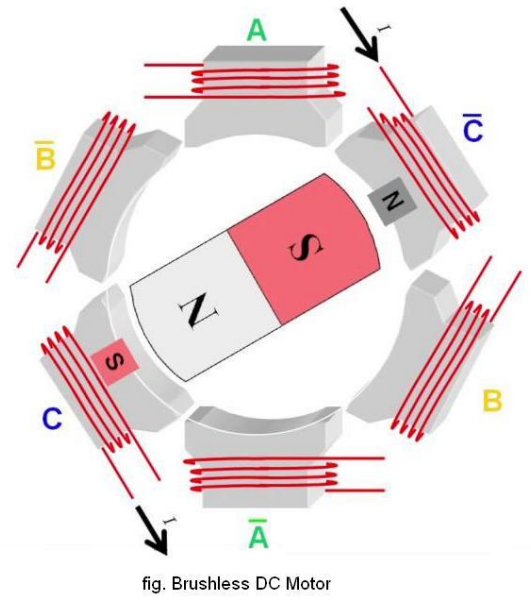

Fig.3 Brushless DC motor

The attractive forces shown in the depiction drives the rotor shaft to turn in clockwise direction. But practically the torque generated through this is insufficient and this cannot be used for heavy duty applications. To achieve good torque characteristics, the stator operation can be promoted to next level by simply energizing the windings in such a way that it repels the permanent magnet and these repulsive forces acts in addition to attractive forces to drive the rotor shaft. This can be done by determining the rotor magnet position and exciting the preceding windings such that it repels the nearby magnet.

From the working principle we can clearly infer the following statements

1. The speed of BLDC motor is controlled by the frequency of commutation i.e., by increasing the switching frequency the speed of motor can be increased and vice-versa.

2. The speed of the motor is also be affected by the force with which the stator attracts and repels the rotor. In other words, if these forces are high then stator attracts the magnetic rotor quickly. So in order to achieve good velocity characteristics the strength of forces should be taken into consideration.

3. The strength of attraction and repulsion between stator and rotor depends on the input to the windings.

4. The input to BLDC motor is DC supply but when it is operated as generator, the output is purely AC. So proper electronic setup is necessary to convert and store the output energy.

Some of the advantages of Brushless DC motor are:

1. High power density, high torque to inertia ratio and high dynamic response due to the small size, low weight.

2. Long operating life and high reliability due to the absence of brushes and metallic commutators.

High speed operation in excess of $80,000 \mathrm{rpm}$ is possible, since these motors are electronically commutated and are not subjected to the limitations of conventional commutations.

\section{Modifications And Operation}

In the above context it is clear that separate equipment is installed for commercial HEVs in process of applying the hybrid technology. But the overall weight of the vehicle is proportionately increased. To account for the relatively best design, the flywheel of the automobile can be made to act as generator/motor whenever required.

This can be achieved by simply redesigning the flywheel of traditional vehicle and making it to act as a rotor in BLDC motor. To make it act as a rotor it should have a magnetic north and south pole as shown in fig below. 


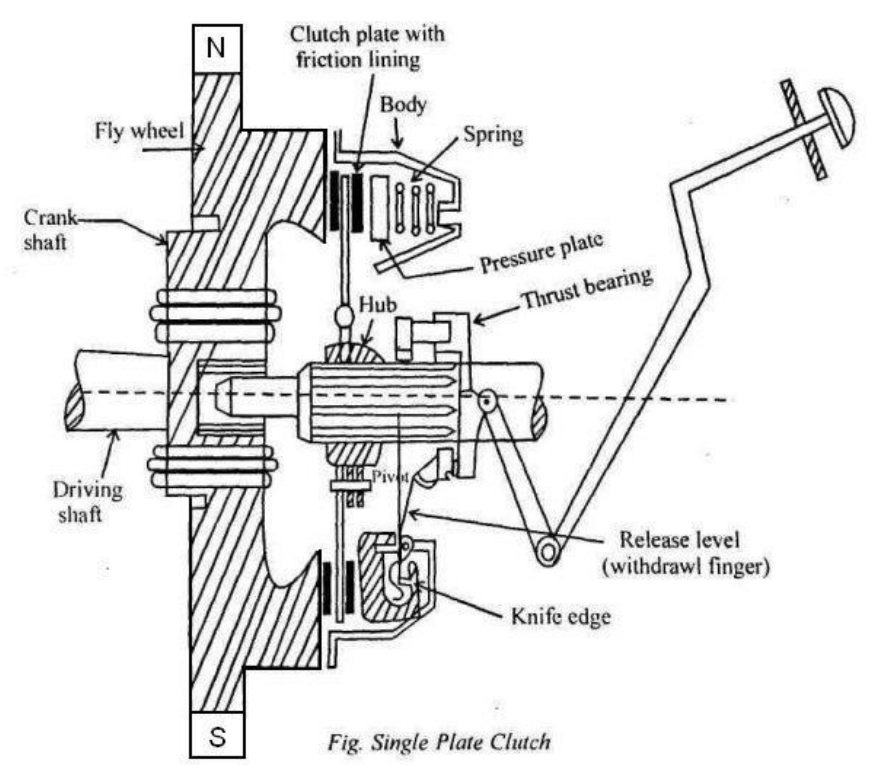

Fig.4 Redesigned flywheel model

The magnetic north and south on the flywheel can be best achieved by using the phenomena of electromagnets. This phenomenon has an advantage of providing high magnetic pole strength with reduced resulting weight due to this arrangement. Alternatively for heavy vehicles the weight of the flywheel is normally high so permanent magnets can be attached feasibly. Around this flywheel the stator winding can be arranged as portrayed in the principle of operation section.

\section{Charging Of Battery:}

When vehicle is powered through internal combustion, the flywheel is always in motion irrespective that vehicle is cruising or idling. During this operation the magnets attached to flywheel rotates and induces voltage on stator winding consequently generating voltage across stator windings and acts as a generator. This generated voltage is purely AC but to store the supply battery are employed which are only powered through DC, so in order to store the generated output electronic converters are used. Electronic converters, converts the AC into proportional DC and this DC can be stored by charging the battery.

In most cases the voltage limiting circuits are arranged to reduce the load on batteries during high accelerations. Additionally electronic control circuits and indicating circuits can be arranged so that the battery charging status can be known to the driver. And control circuit is used to manage the operation of charging or discharging of batteries i.e., to act as generator or motor.

\section{Powering Vehicle through Motor:}

When the arrangement is made to act as BLDC motor, the charge from the battery is given as input to the windings and forcing it to act as motor. In this mode of operation the IC engine is shut down and the whole vehicle is powered by the motor. While discharging, input to the stator winding is purely DC so battery circuit can be directly connected to the windings. Moreover a transformer can be used to step up the voltage and thus increasing the intensity of voltage to windings and increasing strength of attraction or repulsion for rapid movement of rotor.

The conventional throttling action of the driver can be attained by varying the switching frequency of energizing the windings. When switching frequency of energizing the windings the speed of rotation increases and also works contrariwise.

Using this, the starting motor can also be eliminated and BLDC flywheel operation can be used for starting of IC engine also. And no additional mechanism is required to engage transmission, gear box of vehicle with the motor.

Moreover all the advantageous technologies used by commercial HEVs like regenerative braking, auto start/shutdown can be conveniently used through this design also. 


\section{Conclusion And Discussion}

The above discussed method has few challenges to overcome while operation and during the design stage which are analyzed below.

- Installing of magnets on the flywheel would result in various balancing problems. In order to account for perfect balancing the mass on periphery of the magnets attached flywheel should be even so that center of mass of the whole flywheel should be at its geometric center. Because flywheel rotates about its center, any small unevenness in mass distribution will lead in failed operation.

- When acting as a generator, emf is also induced on the flywheel and propagates through crankshaft if the material of flywheel is conductive and this might result in pre-ignition of charge in combustion chamber.

- In this approach the battery is completely drained when operated as motor, so in gasoline powered engines no charge would be left in the battery for spark plugs. So a secondary battery should be kept as contingency or an electronic circuit can be designed to warn when charge in battery falls below required level.

- The permanent magnets attached to the flywheel during operation are subjected to greater centrifugal force. This imparts high pulling force on magnets and may even fail during operation.

The substitute approach to drive HEV is a high potential eco-friendly and efficient technique. It can be implemented for both light and heavy duty applications and substantial reduction in fuel consumption thus reducing the harmful emissions into the atmosphere.

\section{Journal Papers:}

\section{References}

[1]. Caiying Shen et al, "A Comprehensive Overview of Hybrid Electric Vehicles", Article in International Journal of Vehicular Technology, Volume 2011, Article ID 571683, Issue:11-09-2011.

[2]. Young-Joo Lee, Alireza Khaligh, Ali Emadi, "Advanced Integrated Bidirectional AC/DC and DC/DC Converter for Plug-In Hybrid Electric Vehicles", Published in IEEE Transaction on Vehicular Technology, Vol.58, Issue:8-10-2009

[3]. A.Brahma, Y.Guezennec, G.Rizzoni, "Optimal Energy Management In Series Hybrid Electric Vehicles", Proceeding of American Control Conference,2000, Issue:28-06-2000

[4]. Pavan R.S, “A Review On Hybrid Vehicles”, Published in Impact: International Journal of Research in Engineering \& Technology, Vol.2,Issue:05-05-2014 